\title{
Bayesian Inference for Data Assimilation using Least-Squares Finite Element Methods
}

\author{
Richard P. Dwight \\ Assistant Professor, Aerodynamics Group, Faculty of Aerospace, TU Delft, P.O. Box 5058, \\ 2600GB Delft, The Netherlands. \\ E-mail: r.p.dwight@tudelft.nl
}

\begin{abstract}
It has recently been observed that Least-Squares Finite Element methods (LSFEMs) can be used to assimilate experimental data into approximations of PDEs in a natural way, as shown by Heyes et al. in the case of incompressible Navier-Stokes flow [1]. The approach was shown to be effective without regularization terms, and can handle substantial noise in the experimental data without filtering. Of great practical importance is that - unlike other data assimilation techniques - it is not significantly more expensive than a single physical simulation. However the method as presented so far in the literature is not set in the context of an inverse problem framework, so that for example the meaning of the final result is unclear. In this paper it is shown that the method can be interpreted as finding a maximum a posteriori (MAP) estimator in a Bayesian approach to data assimilation, with normally distributed observational noise, and a Bayesian prior based on an appropriate norm of the governing equations. In this setting the method may be seen to have several desirable properties: most importantly discretization and modelling error in the simulation code does not affect the solution in limit of complete experimental information, so these errors do not have to be modelled statistically. Also the Bayesian interpretation better justifies the choice of the method, and some useful generalizations become apparent. The technique is applied to incompressible Navier-Stokes flow in a pipe with added velocity data, where its effectiveness, robustness to noise, and application to inverse problems is demonstrated.
\end{abstract}

\section{Introduction}

Data assimilation has long been of importance in atmospheric science and geophysics, where computational models are largely empirical, or model parameters are inaccessible to measurement. In engineering on the other hand, we are motivated by the complementary errors and limitations of simulations and experiments. The particular example considered in this article is illustrative: using the particle image velocimetry (PIV) measurement technique the velocity of a fluid can be measured; pairs of photos are taken (with a small time increment) of particles transported by the flow, the particles having been illuminated with laser light sheets [2]. Compared to other measurement techniques an extremely large amount of data is returned, but only in a limited 2d-planar "window" of a flow field. This window can not be too close to walls, otherwise laser reflections pollute the images, it is further limited by optical access to the flow, camera resolution, and many other practical considerations. Important quantities such as the pressure are inaccessible. Measurement errors are approximately Gaussian in distribution. On the other hand a Navier-Stokes numerical simulation can encompass the entire 3d flow field in all state variables, giving a complete picture of the flow, but includes discretization error, 
and more importantly modeling of physics (e.g. turbulence), which introduces an unknown bias in the results - it is therefore less trustworthy than experimental observations. Integrating experimental data into a simulation would allow: from a simulation point-of-view, an estimation and reduction of model error, and from an experimental point-of-view, an extension of measured results to the full flow field and all variables. It is our contention that an efficient and robust framework combining the two, would be of considerably more value than either technique in isolation.

Data assimilation techniques can be broadly categorized into methods based on estimation theory, control theory, and stochastic approaches [3], though there are substantial overlaps between these categories. The central tool in estimation theory is Bayesian inference, with which all available knowledge about a system is combined. This reliability of each piece of knowledge is represented by probability measures, and Bayes' theorem is used to determine the posterior probability of the observed data given the model (see Section 3). The posterior may be considered the solution to the data assimilation problem, and question is then how to extract information from the posterior, which is a probability measure on a very high-dimensional space. Proven techniques are the Kalman filter and ensemble Kalman filter, both of which assume a linear model and multivariate normal distributions. Nudging (also called the Newtonian relaxation scheme), relaxes the the simulation towards the observations by adding terms proportional to the difference between the data and the model state to the model residual.

Control theory approaches to data assimilation are typified by the use of an adjoint method to impose the governing equations as a strong constraint on the solution. The cost function is commonly a sum of penalties of initial conditions, model parameters, and data-model mismatch, each weighted with the a priori covariance of the uncertainty or measurement noise. The adjoint and forward simulation can then be solved simultaneously in a coupled iteration, or standard optimization techniques, such as conjugate gradients may be used, with the adjoint providing the gradients. The effort required is at least several times the cost of a single simulation. Most recently stochastic methods have become more popular, in which a stochastic PDE is used to model a conditional probability density equation. However standard solution methods employ Monte-Carlo sampling of the stochastic space, making the cost many hundreds of times that of a basic computation.

The particular technique considered in this article is the LS-FEM data assimilation approach recently proposed by Heyes et al. [1]. Given a system of PDEs, LS-FEM methods define a scalar functional whose minimum is the solution of the system; as opposed to the conventional finite element/finite volume approach of finding a zero of a suitably defined residual. Due to this minimization structure ill-posed systems may be treated readily, and terms may be added to the functional to enforce weak constraints. It is this latter feature that is used in [1], in which terms are added which penalize the difference between the numerical solution and specified experimental data. Thus a data assimilation method is obtained which costs little more than a standard LS-FEM calculation. In numerical experiments it is seen to effectively combine simulation and experimental observations, without regularization terms, in the presence of observational noise.

The method appears to work so well that it is worth asking, why? The theory of LS-FEMs alone offers few insights into this question. The contribution of this article is to explain the behaviour and performance of the method in terms of Bayesian inference. In this context a statistical interpretation of the terms, weighting coefficients and solution can be made, giving a theoretical foundation and practical guidelines for application of the technique.

This article is structured as follows: first the LS-FEM data assimilation approach of [1] is concisely presented in Section 2. In Section 3 the techniques of Bayesian inference are presented in an abstract fashion, together with a discussion of practical considerations when applying them to complex simulation codes. The interpretation of LS-FEM data assimilation as a Bayesian data 
assimilation technique is then described. Section 4 completes the presentation with numerical examples of the method applied to Navier-Stokes flow with added velocity data.

\section{Data assimilation with LS-FEM}

The basic idea is straightforward: given a (first-order) system of partial differential equations on the bounded domain $\Omega \subset \mathbb{R}^{d}$ with piecewise smooth boundary $\Gamma$,

$$
\begin{array}{ll}
A \mathbf{w}=\mathbf{f} & \text { in } \Omega \\
B \mathbf{w}=\mathbf{g} & \text { on } \Gamma
\end{array}
$$

where $A$ and $B$ are linear(ized) differential operators,

$$
A \mathbf{w}:=\sum_{i=1}^{d} A_{i} \frac{\partial \mathbf{w}}{\partial x_{i}}+A_{0} \mathbf{w},
$$

and where $A_{0}, \ldots, A_{d}$ are $N_{\text {eq }} \times N_{\text {var }}$ matrices, and similarly for $B$. Further $\mathbf{f} \in \mathcal{X}(\Omega), \mathbf{g} \in \mathcal{Y}(\Gamma)$ are known functions, and $\mathbf{w} \in \mathcal{W}(\Omega)$ the unknown solution. The standard least-squares solution of this in general overdetermined system is as follows [4]: define a least-squares functional

$$
\mathcal{J}(\mathbf{w} ; \mathbf{f}, \mathbf{g}):=\alpha_{1}\|A \mathbf{w}-\mathbf{f}\|_{\mathcal{X}}^{2}+\alpha_{2}\|B \mathbf{w}-\mathbf{g}\|_{\mathcal{Y}}^{2},
$$

and solve the resulting optimization problem

$$
\mathbf{w}_{\mathrm{LS}}:=\underset{\mathbf{w} \in \mathcal{W}}{\operatorname{argmin}} \mathcal{J}(\mathbf{w} ; \mathbf{f}, \mathbf{g}),
$$

where the spaces and associated norms have been chosen such that the minimizer of the leastsquares functional $\mathcal{J}$ is the same as the solution of (1)-(2), supposing the latter are well-posed. The weighting factors $\alpha_{1}, \alpha_{2}$ allow control of the relative importance of the equations, which may strongly affect the quality of the resulting discretization even in the case of well-posed governing equations. The minimization problem (4) is solved using the calculus of variations to obtain an equivalent variational statement, and subsequently discretization is performed by replacing $\mathcal{W}$ with a finite element space $\mathcal{W}_{h} \subset \mathcal{W}$, chosen to confer desirable properties on the resulting LS-FEM method. Behind the last sentence sits an entire field of research, for which there is no space here. We refer the reader to Jiang [4], and Bochev \& Gunzburger [5] and the references therein.

Now, if some additional information is available regarding the solution (for example from experimental data), it seems reasonable to augment the least-squares functional $\mathcal{J}$ with terms that penalize the difference between the least-squares solution and that data. For example if the measurements are $\mathbf{d}$, taken of a quantity which may be computed from $\mathbf{w}$ with an observation operator $G(\mathbf{w})$, then one possibility is to modify $\mathcal{J}$ as

$$
\tilde{\mathcal{J}}(\mathbf{w} ; \mathbf{f}, \mathbf{g}, \mathbf{d}):=\mathcal{J}(\mathbf{w} ; \mathbf{f}, \mathbf{g})+\alpha_{3}|\mathbf{d}-G(\mathbf{w})|^{2},
$$

where $|\cdot|^{2}$ is the Euclidean norm, and expect to obtain a solution $\mathbf{w}$ that represents some compromise (in a weighted least-squares sense) between the solution of the PDE, which may contain some physical modelling and discretization error, and experiment, with associated measurement error. The minimization of $\tilde{\mathcal{J}}$ can be performed in an identical way to that of $\mathcal{J}$, and as such the additional cost of adding data is negligible.

Note that for standard finite element methods no such simple modification for adding information exists, as the well-posedness of the system is difficult to preserve. Least-squares 
methods on the other hand seem to be a natural choice for data assimilation problems. Current work leaves several questions open however. Most importantly the choice of norm and weighting factor for the data term in (5) is $a d-h o c$, and the significance of the final result is unclear. In [1] the authors treat the simplified version of the data assimilation problem in which the experimental data is known on an internal boundary $\Gamma_{v}$ of $\Omega$, allowing them to apply wellunderstood least-squares boundary treatments to the experimental data as well as to the boundary conditions resulting from the PDE. They weight the experimental term in inverse proportion to the variance of the measurement error in accordance with standard regression theory. Most experimental results take the form of point or integral values however, and here we treat this case. However, our main goal is to achieve insight into the meaning of (5), its weighting, and its minimizer. This will be possible using the Bayesian framework as shown in the following section.

\section{Data assimilation within a Bayesian framework}

To develop a Bayesian interpretation of the LS-FEM data-assimilation method, we begin with an abstract description of Bayesian inference, and discuss practical issues arising in its application to complex simulation codes. Consider the problem:

Problem 3.1 (Data Assimilation) Find parameters $\mathbf{m} \in \mathcal{M}$, where $\mathcal{M}$ may be a finite- or infinite-dimensional space, such that

$$
d_{k}=G\left(\mathbf{m} ; x_{k}\right) \text { for coordinates }\left\{x_{k}\right\}_{k=1}^{K},
$$

where $G$ is an observation operator, $d_{k}$ are observed data, and $x_{k}$ are known coordinates.

Problem 3.1 is ill-posed in general, in particular the model (6) does not take into account noise in the data. A more appropriate model for the observation/simulation system is of the form (dropping the subscript, so that $\mathbf{d}=\left\{d_{k}\right\}_{k=1}^{K}$ etc.):

$$
\mathbf{d}=G(\mathbf{m} ; \mathbf{x})+\boldsymbol{\delta}
$$

where $\boldsymbol{\delta}$ are random variables representing the observational noise, whose statistical properties are known to us, but whose particular value is unknown. For example a measurement instrument or technique might specify (in the ideal case) a probability density function (PDF) for the true value, given a particular instrument reading; this PDF could be used as a definition of $\boldsymbol{\delta}$. More realistically, instruments typically specify a tolerance $\pm \epsilon$, given which one reasonable choice of model for $\boldsymbol{\delta}$ would be a normal distribution with zero mean (unbiased measurements), and standard deviation $\epsilon / 2$ (so that $95 \%$ of errors lie within the tolerance) ${ }^{1}$.

In the setting of the probabilistic model (7), it is natural to adopt a Bayesian approach to Problem $3.1[6,7]$. In Bayesian inference we first encode our prior beliefs about $\mathbf{m}$ (in the absence of data) in the form of a probability density function $\pi_{0}(\mathbf{m})$ - this is known as the prior on $\mathbf{m}$. We then define the data likelihood of obtaining the observed data given a particular $\mathbf{m}$, which in the case of model (7) is simply

$$
\rho(\mathbf{d} \mid \mathbf{m}):=\rho(\mathbf{d}-G(\mathbf{m} ; \mathbf{x})),
$$

where $\rho$ is the density of the random variable $\boldsymbol{\delta}$. Bayes formula relates the prior and likelihood to a posterior probability measure with density $\pi^{\mathbf{d}}(\mathbf{m})$ describing the probability of $\mathbf{m}$ given $\mathbf{d}$ :

$$
\pi^{\mathbf{d}}(\mathbf{m})=\frac{\rho(\mathbf{d} \mid \mathbf{m}) \pi_{0}(\mathbf{m})}{\int_{\mathcal{M}} \rho(\mathbf{d} \mid \mathbf{m}) \pi_{0}(\mathbf{m}) \mathrm{d} \mathbf{m}} .
$$

1 Though a Gaussian density for observational noise does not take into account the possibility of blunders in the data, for which a heavy-tailed distribution such as a Cauchy distribution may be more appropriate. 
or without the normalizing constant:

$$
\pi^{\mathbf{d}}(\mathbf{m}) \propto \rho(\mathbf{d}-G(\mathbf{m} ; \mathbf{x})) \pi_{0}(\mathbf{m}) .
$$

The density $\pi^{\mathbf{d}}(\mathbf{m})$ is the complete solution to Problem 3.1 in the Bayesian setting ${ }^{2}$.

A typical application of this Bayesian framework to a data assimilation problem, is to take $\mathbf{m}$ to be a vector of model parameters $\boldsymbol{\xi}$ of a complex simulation code - for example in an aerodynamics problem, viscosity, Mach number, and turbulence parameters might be considered uncertain, and the code might be a Reynolds-Averaged Navier-Stokes (RANS) tool. In this case the observation operator $G(\boldsymbol{\xi})$ is composed of solving the RANS equations for the solution field $\mathbf{w}$ subject to the parameters $\boldsymbol{\xi}$, and extracting the required observation from $\mathbf{w}$ :

$$
G(\boldsymbol{\xi}):=G(\mathbf{w}(\boldsymbol{\xi}), \boldsymbol{\xi}), \text { subject to } N(\mathbf{w}(\boldsymbol{\xi}) ; \boldsymbol{\xi})=0,
$$

where $N(\mathbf{w} ; \boldsymbol{\xi}):=f(\boldsymbol{\xi})-A(\boldsymbol{\xi}) \mathbf{w}$ is the residual of the RANS code. The posterior density may be interrogated to find more realistic values and uncertainties for the model parameters $\boldsymbol{\xi}$. For finite dimensional $\boldsymbol{\xi}$ of relatively low dimension, this is a tractable problem either using MarkovChain Monte-Carlo (MCMC) methods to sample the posterior density [8], or using optimization procedures to find the maximum of the posterior density, the maximum a posteriori (MAP) estimator for $\boldsymbol{\xi}$.

In this setup the observation operator $G$ defined in (10) is clearly extremely complex, containing a complete simulation code $N$. This high complexity of $G$ is a problem for the accuracy of our statistical model. So far it has been assumed implicitly by the choice of model (7) that $G$ is known and free from error, whereas if it includes a simulation code this is certainly not true. In this case, a more realistic model of the system would be:

$$
\mathbf{d}=G(\mathbf{m} ; \mathbf{x})+\boldsymbol{\delta}+\varepsilon,
$$

where $\varepsilon$ is a random variable encapsulating the error made in modelling $G$. Lumping $\boldsymbol{\delta}$ and $\varepsilon$ together is tempting, as they are both random variables, but unjustified and dangerous. The character of measurement error and (simulation) modelling errors are quite different: the latter will certainly not be mean zero, whereas the former typically will, leading to a bias in the results. The error from the simulation will also often strongly depend on $\mathbf{w}$, whereas observational noise is typically less sensitive.

Modelling simulation error is a difficult problem in which there has been a lot of recent interest. One approach assumes a distribution family for $\varepsilon$, for example a Gaussian, with unknown mean and variance $\boldsymbol{\zeta}=\left\{\mu_{\zeta}, \sigma_{\zeta}^{2}\right\}$, and treats $\boldsymbol{\zeta}$ as additional components of $\mathbf{w}$ to be determined. This hyper-parameter modelling technique has been investigated by a variety of authors $[14,15,16]$, and offers the fascinating potential for characterizing and quantifying modelling error in simulation codes.

\subsection{The LS-FEM Functional as a Bayesian Prior}

In this paper we pursue a quite different approach to dealing with possible modelling errors in $G$ : we reduce the complexity of $G$, by taking $\mathbf{m}:=\mathbf{w}$ to be the entire solution field in all variables, i.e. the $\mathbf{w}$ of Section 2, or in the discrete case all DoFs of the system. Given the complete solution of the governing equations as input, the observation operator becomes a straightforward mapping from the quantities modelled to those observed. This is a trivial

${ }^{2}$ In the case that $\mathcal{W}$ is an infinite-dimensional function space, the integral in (8) is not defined, and Bayes' theorem must be recast in terms of Radon-Nikodym derivatives of probability measures, see [7]. 
operation in the common situation that the latter are a subset of the former (as is the case for the application in Section 4); then $G$ is simply

$$
G(\mathbf{w}):=\mathbf{w}(\mathbf{x}) .
$$

In many other situations $G$ could be implemented with negligible error. The term $\varepsilon$ is therefore negligible, and must no longer be modelled. The complex simulation code, which potentially contains significant errors, is relegated to the prior, where it cannot bias the observations.

By choosing a prior which is large when w satisfies the governing equations "well", and small otherwise, we are encoding our belief (in the absence of observations) that the simulation code is a reasonable model for observations. This statement, which an engineer makes implicitly when she applies a simulation tool to a problem and expects to get a reasonable result, is thereby formalized in the Bayesian setting. The disadvantage of this very natural treatment is that the unknown $\mathbf{m}=\mathbf{w}$ is of infinite dimension (before discretization), or merely extremely high dimension (after discretization), and it is in general a hard problem to obtain information from a probability measure in high dimensions. For example the techniques for sampling the posterior mentioned in Section 3 will become extremely costly.

It is therefore necessary to make a simplifying assumption in the interests of obtaining a practical algorithm. At this point we assume a particular form for the likelihood and prior. The former we assume to be normally distributed with covariance matrix $\Sigma_{\mathbf{d}}, \boldsymbol{\delta} \sim \mathcal{N}\left(0, \Sigma_{\mathbf{d}}\right)$, the latter is taken to be of the form

$$
\pi_{0}(\mathbf{w}) \propto \exp \left\{-\frac{1}{2} \mathcal{J}(\mathbf{w} ; \mathbf{f}, \mathbf{g})\right\}=\exp \left\{-\frac{\alpha_{1}}{2}\|A \mathbf{w}-\mathbf{f}\|_{\mathcal{X}}^{2}-\frac{\alpha_{2}}{2}\|B \mathbf{w}-\mathbf{g}\|_{\mathcal{Y}}^{2}\right\},
$$

using the notation of Section 2. Applying Bayes' theorem (9) we have

$$
\begin{aligned}
\pi^{\mathbf{d}}(\mathbf{w}) & \propto \exp \left\{-\frac{1}{2}\left|\Sigma_{\mathbf{d}}^{-\frac{1}{2}}(\mathbf{d}-G(\mathbf{w}, \mathbf{x}))\right|^{2}-\frac{1}{2} \mathcal{J}(\mathbf{w} ; \mathbf{f}, \mathbf{g})\right\} \\
& =\exp \left\{-\frac{1}{2}|\mathbf{d}-G(\mathbf{w}, \mathbf{x})|_{\Sigma_{\mathbf{d}}}^{2}-\frac{1}{2} \mathcal{J}(\mathbf{w} ; \mathbf{f}, \mathbf{g})\right\},
\end{aligned}
$$

where $|\cdot|_{\Sigma}=\left|\Sigma^{-\frac{1}{2}} \cdot\right|$. Finally we choose not to evaluate the full posterior, but confine ourselves to finding the maximum a posteriori (MAP) estimator, i.e.

$$
\underset{\mathbf{w} \in \mathcal{W}}{\operatorname{argmax}} \pi^{\mathbf{d}}(\mathbf{w})=\underset{\mathbf{w} \in \mathcal{W}}{\operatorname{argmin}}\left\{\frac{1}{2}|\mathbf{d}-G(\mathbf{w}, \mathbf{x})|_{\Sigma_{\mathbf{d}}}^{2}+\frac{1}{2} \mathcal{J}(\mathbf{w} ; \mathbf{f}, \mathbf{g})\right\}
$$

and the relation to the method (5) of Section 2 is clear.

The Bayesian derivation of this formula gives us several insights. Foremost is the interpretation of the simulation code as a prior, and the data as a correcting likelihood with a Gaussian observational noise. This places simulation in role in which it can always be trumped by experiment, which is an appropriate situation (in the absence of experimental blundering). The more experimental data is available, the less part simulation plays in the final result, and the presence of error-free data constrains the simulation to match it exactly. In the complete absence of data the best guess is the simulation result. These features would seem to be eminently compatible with modern scientific philosophy, and are made possible by treating the simulation as a Bayesian prior.

The Bayesian formulation also highlights the meaning of the weighting factors. Crosscorrelation in observational noise, modelled by $\Sigma_{\mathbf{d}}$, arises as an natural generalization of independent noise. The weighting of the prior is more delicate, and it is unclear exactly how $\alpha_{1}$ and $\alpha_{2}$ should be chosen, see Section 3.2 for more. 
Of great practical importance is that solving for a MAP estimator with this method is not significantly more expensive than a single physical simulation, which would appear to be the minimum possible effort when performing assimilation of data into a simulation. Most competing techniques involve some iteration, with the physics code as a component in an optimization or other loop [8], limiting their applicability. By demonstrating that the LS-FEM data assimilation method has Bayesian roots, it is made comparable to them.

\subsection{The LS-FEM Prior as Solution Error}

Finally we mention an alternative interpretation of our choice of prior, which applies under certain conditions on the LS-FEM. Given a compliant discrete LS-FEM with discrete space $\mathcal{W}_{h}$, the residual norm is an estimate for the discretization error. Specifically, using the notation of Section 2, and neglecting boundary terms for conciseness, we have

$$
\left\|\mathbf{w}_{h}-\tilde{\mathbf{w}}\right\|=\left\|A \mathbf{w}_{h}-\mathbf{f}\right\|_{\mathcal{X}}^{2},
$$

where the energy norm is defined as $\|\mathbf{w}\|=\|A \mathbf{w}\|_{\mathcal{X}}^{2}$, and $\tilde{\mathbf{w}}$ is the solution of (1). Alternatively if a result in the solution norm $\|\cdot\|_{\mathcal{W}}$ is preferred, we have the weaker result

$$
c_{1}\left\|\mathbf{w}_{h}-\tilde{\mathbf{w}}\right\|_{\mathcal{W}} \leq\left\|A \mathbf{w}_{h}-\mathbf{f}\right\|_{\mathcal{X}} \leq c_{2}\left\|\mathbf{w}_{h}-\tilde{\mathbf{w}}\right\|_{\mathcal{W}}, \quad \forall \mathbf{w}_{h} \in \mathcal{W}_{h},
$$

where $c_{1}, c_{2}$ are independent of $\mathbf{w}$ and $h$ [5]. In both cases, the equivalence of solution error and LS-FEM functional allow us to interpret the prior in (14) as the distance in some norm of $\mathbf{w}$ from the solution of the governing equations - also an apparently very reasonable choice of prior. The data assimilation problem may be rewritten:

$$
\mathbf{w}:=\underset{\mathbf{w}_{h} \in \mathcal{W}_{h}}{\operatorname{argmin}}\left\{\left|\mathbf{d}-G\left(\mathbf{w}_{h}, \mathbf{x}\right)\right|_{\Sigma}^{2}+\left\|\mathbf{w}_{h}-\tilde{\mathbf{w}}\right\|\right\} .
$$

The final term in (16), involving the distance from a specified solution, is reminiscent of Tikhonov regularization, a common form of regularization used when solving inverse problems with variational methods, however in that context the choice of solution and norm are often somewhat arbitrary $[17,8]$.

\section{Application to Navier-Stokes Flow in a Pipe}

For the numerical demonstration of the method we consider 3d Navier-Stokes fluid flow, and the integration of Eulerian velocity data obtained for example from PIV. The data used in the following examples is generated artificially from a high-resolution numerical solution with added zero mean Gaussian noise of specified variance. Hence strictly speaking modelling error is zero, and only discretization error is present, however for the method order and mesh considered here, the discretization error changes the features of the solution, and as such has the character of modelling error.

The standard first-order velocity-vorticity-pressure formulation of NS is used:

$$
\begin{aligned}
\nabla \times \mathbf{v}-\boldsymbol{\omega} & =0 & & \text { in } \Omega, \\
\sqrt{\operatorname{Re}}(\mathbf{v} \cdot \nabla) \mathbf{v}+\nabla p+\frac{1}{\sqrt{\operatorname{Re}}} \nabla \times \boldsymbol{\omega} & =0 & & \text { in } \Omega, \\
\nabla \cdot \mathbf{v} & =0 & & \text { in } \Omega, \\
\nabla \cdot \boldsymbol{\omega} & =0 & & \text { in } \Omega .
\end{aligned}
$$

where $\boldsymbol{\omega}$ is the vorticity, and the last equation adds no extra information, but modifies the leastsquares functional, penalizing breach of vorticity conservation. Additional we then have $\boldsymbol{\omega} \in H^{1}$ 
rather than $\boldsymbol{\omega} \in H^{1}$ (curl) which would require Nedelec elements for a conformal discretization. The functional uses the $L^{2}$-norm $\|\cdot\|_{0}^{2}$ in order to obtain a practical method, though it results in a functional with no strict $H^{1}$-coercivity. The mass-conservation component is weighted with the constant $c_{m}$. Dirichlet velocity boundary conditions are implemented essentially, by restriction of the discrete solution space. The remaining conditions are added to the LS-FEM functional, with an inverse mesh-spacing weighting. A well-known issue with the $\mathbf{v}-\boldsymbol{\omega}-p$ first-order formulation is that mass is conserved poorly. This mass-loss plays the role of modelling error for demonstrating the technique, though in fact it will diminish to zero as the mesh is refined.

The test-case chosen to demonstrate the effectiveness of the method is similar to that used in [1]. A schematic of the setup is shown in Figure 1. Essentially fluid is injected with a specified velocity profile $\mathbf{v}_{\text {in }}$, into a pipe of square-cross section and transported to the other end. The Reynolds number based on the pipe width and inflow velocity is 100 . The grid used in all calculations has $40 \times 8 \times 8$ cells, and continuous Lagrange tensor-product $Q_{p}$ finite elements are used, whose order $p$ is varied to control the accuracy of the calculation.

A reference solution is plotted in Figure 2. Using $Q_{5}$ elements without reduction of the basis implies $6^{3} \cdot 7$ degrees of freedom (DoFs) per element, for a total of $\sim 3.8 \cdot 10^{6}$ DoFs.

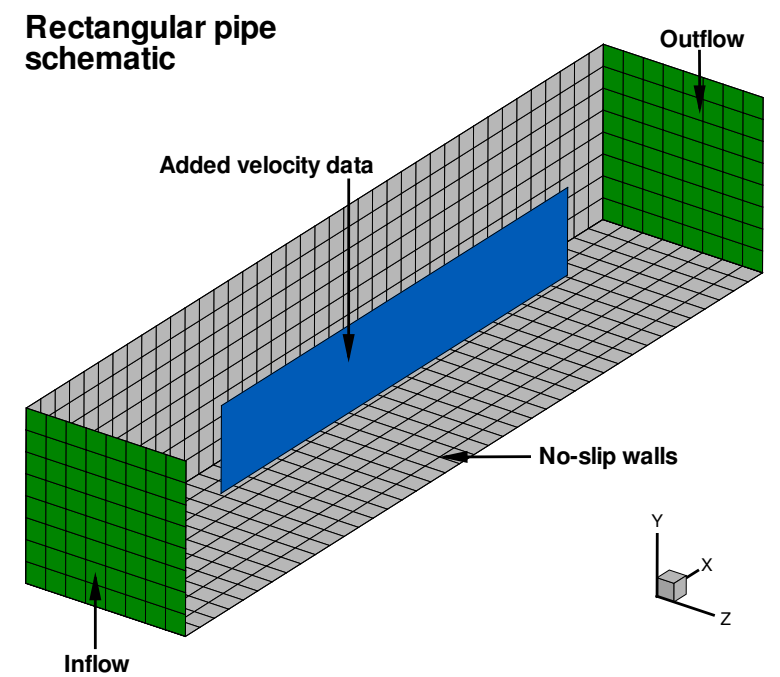

Figure 1. Schematic of the pipe flow. The aspect ratio is $5: 1: 1$, the added velocity data extends from $25 \%-75 \%$ of the pipe in $x$ - and $y$ coordinates, and is centered in $z$ coordinates.

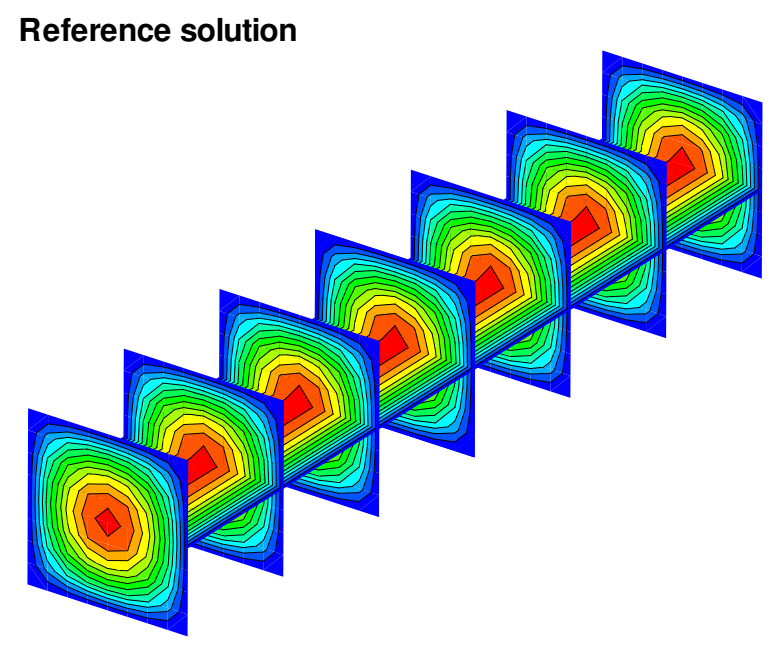

Figure 2. Velocity for reference numerical solution for an imposed quadratic inflow velocity $\mathbf{v}_{\text {in }}=y(1-y) z(1-z)$, obtained with LS-FEM on a $40 \times 8 \times 8$ grid, Lagrange $Q_{5}$ elements, $c_{m}=10$.

To demonstrate the effects of the Bayesian data assimilation it is convenient to start from a low quality numerical solution. This is obtained by using LS-FEM with $Q_{1}$ elements and $c_{m}=1$. The simulation result without added data is shown in Figure 3 where substantial loss of mass is apparent. While it's possible to increase $c_{m}$ to improve mass conservation, this leads to an ill-conditioned system, and what is worse: other equations are necessarily under-weighted - so that the solution is not necessarily improved. Note that we are in no way advocating the use of this numerical formulation with $Q_{1}$ elements in practice, it serves purely as a convenient example of a poor model for data assimilation.

The least-squares functional is modified according to (14), with data added on the plane depicted in Figure 1. Only velocity data in-plane was used, to better simulate the PIV situation, where velocity components normal to the plane of the laser are not captured. Two levels of observational noise were used, and in both cases the correct LS weighting was used and the noise 


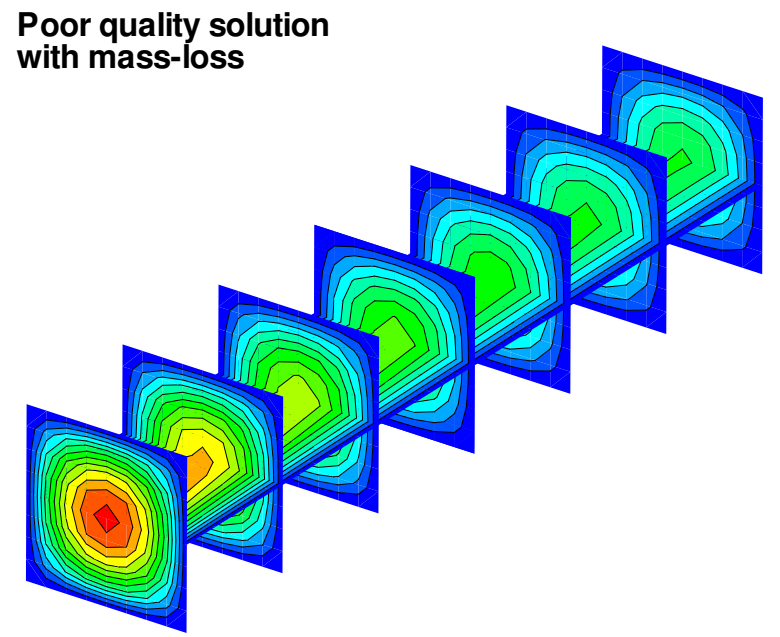

Figure 3. Poor quality numerical solution with significant mass loss, LS-FEM $40 \times 8 \times 8$ grid, $Q_{1}, c_{m}=1$.

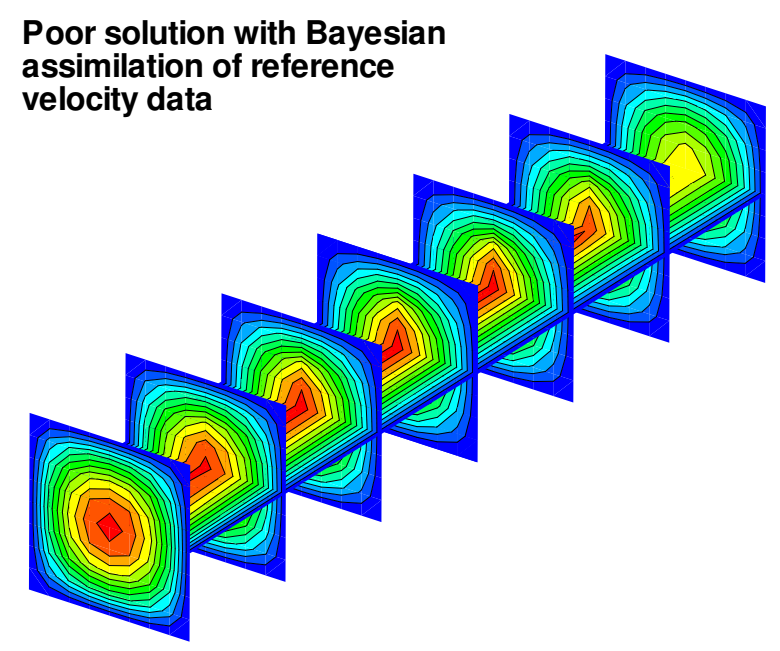

Figure 5. Poor quality solution (LS-FEM $Q_{1}$ ) augmented with velocity data from the reference solution. Data noise $\delta \sim \mathcal{N}(0,0.1 I)$.

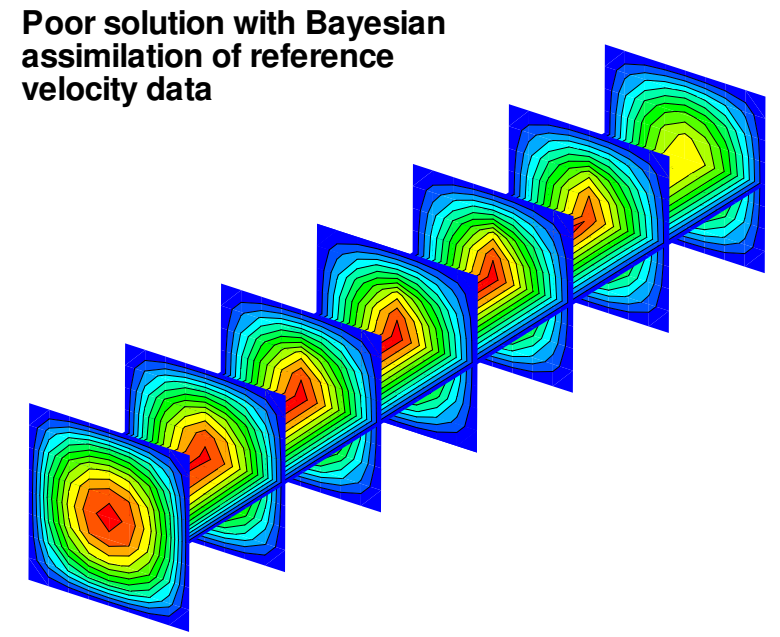

Figure 4. Poor quality solution (LS-FEM $Q_{1}$ ) augmented with velocity data from the reference solution. Data noise $\delta \sim \mathcal{N}(0,0.01 I)$.

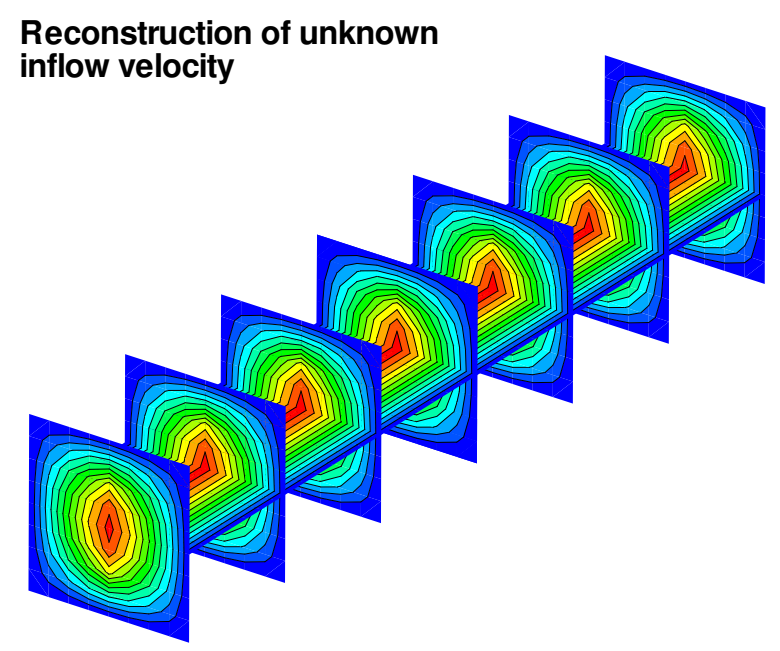

Figure 6. Unspecified inflow condition, velocity reconstructed everywhere from planar velocity data. LS-FEM $Q_{3}, c_{m}=10$.

was assumed to be uncorrelated, $\boldsymbol{\delta} \sim \mathcal{N}(0,0.01 I)$ and $\boldsymbol{\delta} \sim \mathcal{N}(0,0.1 I)$. The velocity profiles are plotted in Figures 4 and 5 respectively. In both cases it can be seen that the numerical solution is improved dramatically, with significantly less loss of mass where the "experimental" data has an influence. Further the numerical solution can be seen to be relatively smooth and reasonable in both cases, despite the high level of noise, such that the higher levels of noise in the second case do not have a visible effect.

As a final demonstration of the robustness and power of the method, we consider the inverse problem obtained by treating the inflow velocity $\mathbf{v}_{\text {in }}$ as unknown, but specifying velocity data on the data plane. The reference velocity data with added noise $\boldsymbol{\delta} \sim \mathcal{N}(0,0.01 I)$ is used on the 
data plane, and $Q_{3}$ elements are used so that the numerical model is reasonably accurate. The result is shown in Figure 6, and is a convincing reconstruction of the features of the reference solution given the available data. No regularization terms were needed and the observational noise seems to present no problem - in fact the only modification of the code necessary was turning off the inflow boundary condition.

\section{Conclusions}

The contribution of this article is a Bayesian formulation of a recently proposed LS-FEM data assimilation technique. By choosing the entire flow solution $\mathbf{w}$ as the statistically modelled quantity, the governing equations can be seen to compose a Bayesian prior, and as a consequence modelling and discretizations errors in the simulation do not affect the accuracy of the model of observational noise. It is therefore not necessary to have complete faith in the simulation code. On the other hand, even with sparse experimental data the method will give a detailed solution field, determined mostly by the simulation code. These properties seem nicely in line with established scientific practice, where experimental data always trumps simulation results, but simulation is the best option in many cases. The LS-FEM framework allows definition of the data/simulation problem without concerns for well-posedness, or stability and robustness of the solution method. The approach is also extremely cheap, when compared with other variational data assimilation techniques. We consider the method to have significant potential for these reasons.

The numerical investigations performed here (and so far in the literature) must be seen as preliminary, and the primary concern for further work should be the application to substantially more complex equations and flows to determine the usefulness of the method in general. The test-case of Section 4 is unfortunately simple in that the inflow velocity profile is transported with little modification to the outflow. In follow-up work we aim to consider applications from our field of interest, applied aerodynamics, and include data from PIV experiments.

\section{References}

[1] Heys J, Manteuffel T, McCormick S, Milano M, Westerdale J and Belohlavek M 2010 Journal of Computational Physics 229 107-118 ISSN 0021-9991

[2] van Oudheusden B and Scarano F 2009 Measurement Science and Technology 20

[3] Robinson A and Lermusiaux P 2000 Overview of data assimilation Physical/Interdisciplinary Ocean Science 62 Harvard University Reports

[4] Jiang B N 1998 The Least-Squares Finite Element Method: Theory and Applications in Computational Fluid Dynamics and Electromagnetics 1st ed (Berlin: Springer)

[5] Bochev P B and Gunzburger M D 2009 Least-Squares Finite Element Methods 1st ed (Applied Mathematical Sciences vol 166) (New York: Springer)

[6] Apte A, Jones C K R T and Stuart A M 2008 Tellus A 60 336-347

[7] Stuart A 2010 Acta Numerica 19

[8] Tarantola A 2004 Inverse Problem Theory and Methods for Model Parameter Estimation (Society for Industrial and Applied Mathematics)

[9] Berndt M, Manteuffel T A and McCormick S F 1997 Electronic Transactions on Numerical Analysis 6 35-43

[10] Becker R and Rannacher R 1998 Proceedings of ENUMATH-97, Heidelberg (Singapore: World Scientific Publishing)

[11] Giles M and Suli E 2002 Acta Numerica, Cambridge University Press 145-236

[12] Dwight R 2007 Proceedings of ICFD Conference, University of Reading

[13] Dwight R 2008 Journal of Computational Physics 227 2845-2863 dOI: 10.1016/j.jcp.2007.11.020

[14] Kaipio J and Somersalo E 2005 Statistical and Computational Inverse Problems (Applied Mathematical Sciences vol 160) (Springer)

[15] Kaipio J and Somersalo E 2007 Journal of Compuational and Applied Mathematics $198493-504$

[16] Christie M 2010 Simplicity, Complexity and Modelling (New York: John Wiley)

[17] Engl H, Hanke M and Neubauer A 1996 Regularization of Inverse Problems (Kluwer)

[18] Heys J, Lee E, Manteuffel T and McCormick S 1997 Journal of Computational Physics 226 994-1006 